\title{
Acquisition and forgetting of information in long-term memory as a function of certain hierarchical structure variables*
}

\author{
KENNETH A. DEFFENBACHER \\ University of Nebraska, Omaha, Nebraska 68101 \\ and \\ JOHN G. MISCIK and JERRY JAROMBEK \\ Human Engineering Laboratory, Aberdeen Proving Ground, Maryland 21005
}

\begin{abstract}
Ninety community college students learned one of three types of hierarchically organized information presented either as a graphical representation of the hierarchy or as an ordered list of one-directional connections derived trom top to bottom in the hierarchy. Three weeks later, Ss were tested for free recall, cued recall, and relearning. Major findings were: (a) For all three hierarchy types acquisition was much better with graphical rather than list representation of the hierarchy. (b) All three retention measures yielded no evidence that a graphical representation was a superior retrieval device at 3-week recall.
\end{abstract}

In a recent article Nelson and Smith (1972) examined the effect of certain hierarchical structure variables on acquisition and retention of long-term memory (LTM) information. Their most striking finding was that the mode in which hierarchically organized information is presented has a very pronounced effect on acquisition and a lesser, but nonetheless significant, effect on retention of the information 3 weeks later. When the interitem connections to be learned are presented simultaneously in a graphical representation of the hierarchy, learning and retention are much more efficient than when the same hierarchical organization of information is presented simultaneously as an ordered list of one-directional connections between members of item pairs.

Though Nelson and Smith have offered no explanation of their graphic presentation effect, presumably it depends at least in part on the fact that hierarchical structure may be less easily apprehended when it is presented as an ordered list of associations. Hierarchical relations such as class inclusion are nonspatial continua and when they are mapped onto a spatial continuum like that offered by a graph, their structure can be more easily apprehended. This particular advantage of graphic presentation clearly relates to the popularity of graphs for representing functional relationships in science, as Attneave (1974) has pointed out. Attneave argues that one human means for representing relationships in the brain is an analogue \footnotetext{
*This article may be reproduced in full or in part for any
purpose of the U.S. Government. Special thanks are extended to Evan Brown for valuable discussion. Please address reprint requests to Kenneth A. Deffenbacher, Department of Psychology, University of Nebraska at Omaha, Omaha, Nebraska 68101.
}

model of physical space and that we have a highly developed facility for mapping both spatial and nonspatial relations onto this medium. In this medium, class inclusion relations among verbal items could be represented, analogously, by other relations, spatial ones. Quite possibly, then, hierarchic structure may be less easily apprehended when it has been digitized, as in the case when it has been presented as a serial list of directed associations.

Nelson and Smith apparently have demonstrated the mnemonic advantage of graphic representation for both acquisition into and forgetting from LTM. The explanation offered in the preceding paragraph certainly could account for faster, less error-prone acquisition for Ss in the graphic presentation condition. In addition, however, Nelson and Smith's Stanford undergraduates found graphic representation a more effective retrieval device 3 weeks later. As Nelson and Smith observe, though, acquisition into and forgetting from LTM are theoretically independent processes. Olton (1969), for example, has found that the use of sentence mnemonics facilitated LTM acquisition but had no effect on recall 1 week later. One intent of the present study is to further examine Nelson and Smith's graphic presentation effect on LTM acquisition and forgetting with a $\mathrm{S}$ sample similar in age to theirs but much less selected in terms of its cognitive abilities, community college students. This latter S sample more nearly resembles Olton's public school fifth-grade Ss in terms of relative lack of selection for cognitive abilities.

It should be noted that Nelson and Smith's results were obtained with a single hierarchy type henceforth referred to as an arbitrary hierarchy. Hierarchies of this type would seem to have a minimum of conceptual 
validity for $\mathrm{S}$ in that the hierarchy levels are not labeled and the node labels are not terribly meaningful, single consonants in Nelson and Smith's case. Two additional hierarchy types may be distinguished, a priori and partially a priori. A priori hierarchies are those which already exist as a means for expressing certain semantic and conceptual relationships in a language, for example, the biological classification hierarchies used in the present study or the conceptual hierarchies employed by Bower, Clark, Lesgold, and Winzenz (1969) $;^{1}$ here the hierarchy levels have explicit or implicit labels, and the node labels are concrete nouns or adjectives. Partially a priori hierarchies would seem to represent an intermediate type in terms of the amount of conceptual validity they have for S. Acquisition of a partially a priori hierarchy would not be unlike acquisition in a situation where, for example, a student already knows a biological classification scheme (levels of the hierarchy), but is unfamiliar with the particular organisms involved (nodes of the hierarchy). Hence, hierarchies of this type have meaningful labels for the hierarchy levels, but node labels which are not very meaningful, CVCs in the present study, for example. A further intent of the present study, then, is to determine whether the graphic presentation effect is independent of hierarchy type, that is, independent of the presence or absence of hierarchy level labels and the degree of node label meaningfulness.

\section{METHOD}

Ninety Harford Community College (Maryland) students paid $\$ 8.00$ for their participation were randomly assigned $(n=15)$ to the cells of a 2 (mode of hierarchy presentation: graph or list) by 3 (hierarchy type: a priori, partially a priori, or arbitrary) analysis of variance design.

Sample graphical and list representations of a hierarchy are displayed in Fig. 1. These two hierarchies are two of the six hierarchies employed on warmup trials. The six hierarchies used in the experiment proper involved four rather than three levels and a total of 34 one-directional connections between items rather than seven. Both a priori hierarchies were biological classification schema similar to those in Fig. 1; both partially a priori hierarchies differed from a priori hierarchies only in having CVCs (39\% average association value) as node labels. The two arbitrary hierarchies were exactly the same as those displayed in Nelson and Smith's article, having single consonants as node labels and no labeling of hierarchy levels.

The procedure faithfully mimics that recorded by Nelson and Smith. Ss in the graph conditions were told that every item below a given item "went with" that item; Ss in the list conditions were told that the first item of a given pair "went with" the second item. Prior to learning the main hierarchy, $S$ had two complete warmup trials on a practice hierarchy such as those displayed in Fig. 1. Acquisition was by the study-test procedure, the learning criterion being one errorless test trial. During each 2-min study period, S studied either the complete list or the complete graph from top to bottom. Immediately after study, $\mathrm{S}$ shadowed digits for $20 \mathrm{sec}$ to eliminate subsequent recall from short-term memory (STM). Then $S$ had 4 min to recall as many of the 34 connections as he could by marking the appropriate cells of a test grid. The test grids had all the hierarchy items randomly ordered at the edges of the rows and columns (three versions of each test grid were rotated across test

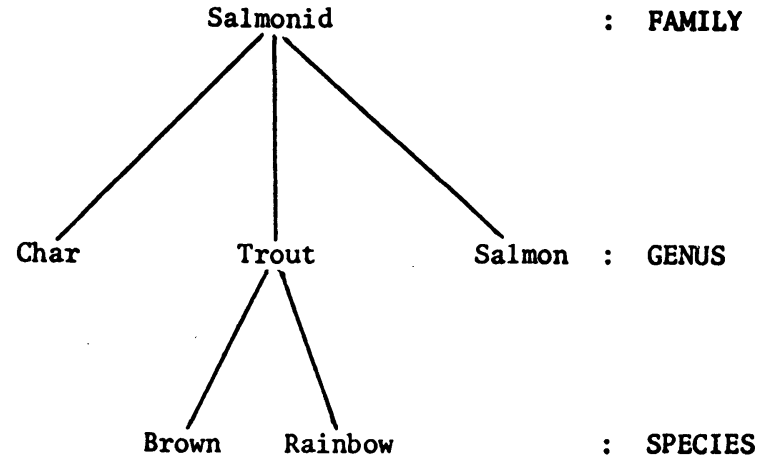

(a)

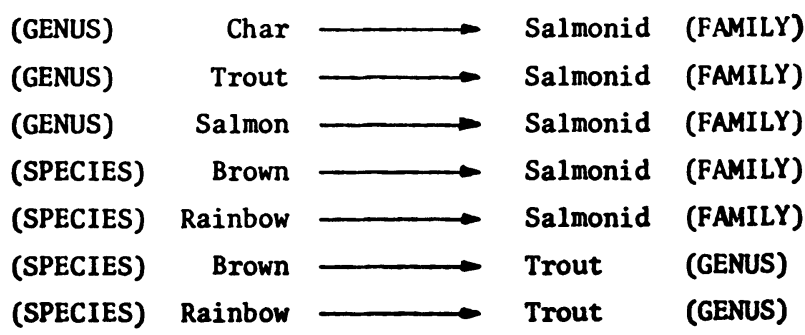

(b)

Fig. 1. (a) Sample graphical representation of an a priori hierarchy (b) Sample list representation of an a priori hierarchy.

trials). The $\mathrm{S}$ was to make a mark in the appropriate cell whenever a given row item "went with" a given column item; S was to make exactly 34 marks even if guessing were required. Following each test trial, S provided himself with $30 \mathrm{sec}$ of feedback by placing a transparent plastic feedback sheet on top of his test grid to determine where he had made errors. The feedback sheet showed a test grid with a black dot in the center of each of the 34 correct cells. When criterion had been reached, no mention was made of any subsequent retention test.

The second session was 3 weeks after the acquisition session. First, $\mathrm{S}$ had a self-paced free-recall test using any form of written recall he wished. He was to write down as many of the associations as he could remember, guessing when unsure, so that the total was 34 . Second, $S$ had a self-paced cued-recall test using the test grid; again 34 marks were to be made. There was no feedback after either of these two retention tests. Finally, $S$ had one relearning trial which followed the same procedure as 3 weeks previously.

\section{RESULTS AND DISCUSSION}

Analyses of variance were performed on five separate measures, acquisition trials and errors, free-recall errors, cued-recall errors, and relearning errors. There were significant effects for mode of hierarchy presentation only in the case of the two acquisition measures (trials: $\mathrm{F}(1,84)=51.46, \mathrm{p}<.001$; errors: $\mathrm{F}(1,84)=49.41$, $\mathrm{p}<.001)$. In each case graphical representation of the hierarchy was superior to list representation; for trials, the means were 3.33 and 5.93 while for errors they were 26.31 and 59.58. As none of the five analyses indicated a significant interaction, it is clear that graphical 
representation facilitated at least acquisition across levels of hierarchy type, a priori, partially a priori, and arbitrary.

For both Nelson and Smith's Stanford undergraduates and the community college Ss of the present study, graphical representation of hierarchies appears to have been a powerful facilitator of acquisition into LTM. The present study further established that this particular graphic representation effect is independent of hierarchy type. However, the present study yielded no evidence that graphical representation facilitated retrieval from later stages of LTM processing. Though this result runs counter to that of Nelson and Smith, it is similar to Olton's finding that a mnemonic device may facilitate acquisition into but not retrieval from LTM. Clearly Ss in the graph conditions of the present study were actually retaining a graphical organization over the 3 -week retention interval in that $93 \%$ of them drew a graph during the free-recall test, a figure comparable to Nelson and Smith's 87\%. Possibly there exists an information processing difference between Stanford undergraduates and at least some community college Ss such that the latter are not able to take advantage of the superior retrieval properties of a graphical representation in the later stages of LTM processing. Hunt (1973), for example, has found information processing differences on laboratory tasks for Ss who have been differentiated on the basis of intelligence. To ascertain whether there were any community college Ss who behaved as did Stanford undergraduates at three weeks, post hoc 2 by 3 analyses of variance were performed on the three retention measures with only the top 1/3 (in terms of fewest acquisition errors) of Ss in each of the original treatment groups. The pattern of results was the same as obtained with the full 15 Ss per cell. Perhaps further researches in the area of memory organization should be more sensitive to the possibility of consistent individual differences in the ability to process certain memory structures.

All analyses except the one of relearning errors yielded significant effects for hierarchy type. For acquisition trials means for a priori, partially a prior, and arbitrary hierarchy types were $3.33,5.70$, and 4.87 $[\mathrm{F}(2,84)=14.63, \mathrm{p}<.001]$; similar means for errors were $28.80,57.07$, and $42.97[\mathrm{~F}(2,84)=11.89$, $\mathrm{p}<.001]$. A series of Newman-Keuls comparisons indicated that for both trials and errors a prior hierarchies facilitated acquisition more than either partially a priori or arbitrary ones $(\mathrm{ps}<.01)$. The error measure also indicated superior performance by the arbitrary hierarchy. condition relative to the partially a priori condition $(\mathrm{p}<.05)$. Freu-recall means for a priori partially a priori, and arbitrary hierarchy types were $16.60,32.63$, and 13.43 , respectively, $[F(2,84)=49.30$, $n<.0011$; similar means for cued recall were 5.77, 10.97 , and $10.33[\mathrm{~F}(2,84)=5.51, \mathrm{p}<.01]$. A
Newman-Keuls series indicated that in free recall a priori and arbitrary hierarchies were each superior to partially a priori ones $(\mathrm{ps}<.01)$ while in cued-recall a priori hierarchies were superior to each of the other two types (ps $<.01$ ).

Not suprisingly, a priori hierarchies generally facilitated LTM acquisition and retention relative to the other two hierarchy types. Less expected, however was the finding that not only did partially a priori hierarchies not facilitate acquisition and retention relative to arbitrary hierarchies (acquisition trials, cued-recall errors), but they actually resulted in inferior performance in two cases (acquisition errors, free-recall errors). One might suppose that if partially a priori hierarchies truly possessed more conceptual validity for $S$ than do arbitrary hierarchies, then at the very least they should not have led to inferior performance in any instance. One possible reason for this unexpected finding might be suggested by some comments volunteered by a number of Ss in postexperimental interviews. Ss in the arbitrary hierarchy groups noted that there was no prior structure, "nothing to go on." To make up for the lack of prior structure Ss in these groups resorted to heavy use of mnemonics of their own choosing, for example, "I let the letters be the first letters of last names of people I knew." Ss in the partially a priori groups, on the other hand, reported that they were more constrained in their development of mnemonics. Not only did the CVCs apparently yield fewer mnemonic possibilities in and of themselves but all mnemonics had to be biological ones. Quite possibly, then, partially a priori hierarchies did indeed possess greater prior structure for Ss in this study than did arbitrary hierarchies, but the greater mnemonic possibilities in the latter case more than compensated for the lack of prior structure.

\section{REFERENCES}

Attneave, F., How do you know? American Psychologist, 1974, 29, 493-499.

Bower, G., Clark, M., Lesgold, A., \& Winzenz, D. Hierarchical retrieval schemes in recall of categorized word lists. Journal of Verbal Learning \& Verbal Behavior, 1969, 8, 323-343.

Hunt, E. Information-processing aspects of intelligence. Paper presented at the meeting of the Midwestern Psychological Association, Chicago, May, 1973.

Nelson, T., \& Smith, RE. Acquisition and forgetting of hierarchically organized information in long-term memory. Journal of Experimental Psychology, 1972, 95, 388-396.

Olton, $R$. The effect of a mnemonic upon the retention of paired-associate verbal material. Journal of Verbal Learning \& Verbal Behavior, 1969, 8, 43-48

\section{NOTE}

1. Though Bower et al employed what are referred to here as a priori hierarchies, they did not manipulate the hierarchical information in the same manner as Nelson and Smith. $R$ ather, they compared acquisition of graphically presented information where the labels of the graph's nodes had been randomly repositioned in the hierarchy with the condition where this had not been done, finding that the latter condition promoted more efficient acquisition into LTM.

(Received for publication August 21, 1974.) 